Proceedings of the 48th International School and Conference on the Physics of Semiconductors "Jaszowiec 2019"

\title{
The Influence of Chromium Thermal-Diffusion-Based Doping on the Spatial Distribution of Luminescence Intensity in ZnSe
}

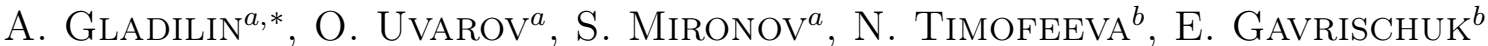 \\ AND V. KALINUSHKIN ${ }^{a}$ \\ ${ }^{a}$ Prokhorov General Physics Institute of the Russian Academy of Sciences, 38 Vavilova Str., 119991 Moscow, Russia \\ ${ }^{b}$ Devyatykh Institute of Chemistry of High-Purity Substances of the Russian Academy of Sciences,
} 49 Tropinina Str., 603950 Nizhny Novgorod, Russia

\begin{abstract}
We have studied the influence of chromium doping of ZnSe crystals at $1250{ }^{\circ} \mathrm{C}$ on the spatial distribution of luminescence in the spectral range of 0.44 to $0.73 \mu \mathrm{m}$. It is expected that several types of defective-impurity centers are formed due to the doping process. Centers of at least one type demonstrate complex distribution and lead to formation of area with high intensity luminescence looking like a bright strip parallel to doping surface.
\end{abstract}

DOI: 10.12693/APhysPolA.136.637

PACS/topics: two photon excitation, luminescence, semiconductors, doping, ZnSe:Cr

\section{Introduction}

The ZnSe crystals doped with $\mathrm{Fe}^{2+}$ and $\mathrm{Cr}^{2+}$ are used as an active medium for solid state laser developed for mid-IR spectral range $(3-5 \mu \mathrm{m})$. Recently, the lasing characteristics of $\mathrm{ZnSe}: \mathrm{Fe}^{2+}$ and $\mathrm{ZnSe}: \mathrm{Cr}^{2+}$ have been improved [1-5]. The medium parameters (optical transmission, absorption, and luminescence) depend on many factors including the dopant and its concentration, as well as on the nature of intrinsic and impurity defects. The lattice imperfections are due to crystal growth conditions, in particular, the influence of high temperature diffusion of dopant ions. The defect control at each preparation stage is significant for optimization of technological routes of laser element production based on $\mathrm{ZnSe}: \mathrm{Fe}^{2+}\left(\mathrm{Cr}^{2+}\right)$.

The high temperature diffusion leads to concentration inhomogeneity in laser crystals. Thus, studying of spatial distributions of different characteristics is of great importance. The two-photon excitation (TPE) microscopy is an efficient method for following spatial distribution luminescence. The method allows recording luminescence spectra inside the crystal volume, thus making possible search for correlation between luminescence variations and the dopant concentration profile $[6,7]$. Previously, we reported investigation of $\mathrm{ZnSe:Fe} \mathrm{[8-10]} \mathrm{based} \mathrm{on} \mathrm{that}$ method. The first results of $\mathrm{ZnSe}: \mathrm{Cr}$ was demonstrated in [8]. Results of the detailed studies of the chromium doped crystals are discussed in the present paper.

\section{Samples and experiment}

The sample investigated were polycrystalline $\mathrm{ZnSe}$ plates doped with chromium by high temperature diffusion. Polycrystalline plates were produced using chemical

\footnotetext{
* corresponding author
}

vapor deposition (CVD) technique. A chromium layer with the thickness of about $1 \mu \mathrm{m}$ was then deposited by electron-beam sputtering. The samples were annealed for $28 \mathrm{~h}$ at $T=1250^{\circ} \mathrm{C}$ and high isostatic pressure (HIP) $P=100 \mathrm{MPa}$. The spatial distribution of luminescence was followed by two-photon excitation (TPE) microscopy using Carl Zeiss 710 NLO confocal microscope equipped with a femtosecond pulsed Ti:sapphire laser operating in the $710-1050 \mathrm{~nm}$ spectral range $[6,7]$. We used the wavelength of $800 \mathrm{~nm}$ in the experiments. The output power was varied in order to achieve contrast images. The setup operated in the $425-725 \mathrm{~nm}$ spectral range and provided up to $3 \mathrm{~nm}$ spectral resolution. In our experiments, we used $10 \mathrm{~nm}$ spectral resolution. The optical setup registered luminescence from the excited volume, which was of about several cubic $\mu \mathrm{m}$. The microscope features made possible shifting the excited volume to the depth down to $2 \mathrm{~mm}$ from the crystal surface. Software used allows obtaining spectral characteristics of each point on the map with the $1 \mu \mathrm{m}$ spatial resolution. The spatially resolved concentration of optically active chromium was determined by absorption measurements at $1.7 \mu \mathrm{m}$ wavelength. The experiment was carried out on a HYPERION 2000 IR microscope equipped with a Tensor-27 IR-Fourier spectrometer (Bruker). The method is described in detail in [11].

The initial undoped samples, previously demonstrated to be high purity polycrystalline CVD-ZnSe [8], were treated in two ways, either doped with chromium with HIP or annealed with HIP without the dopant.

\section{Results and discussion}

Figure 1a shows the spectra at different points, marked at planar map (Fig. 1c) which was obtained at the wavelength of $473 \mathrm{~nm}$ at the depth of $100 \mu \mathrm{m}$ from the surface of CVD-ZnSe:Cr crystal. The results are typical of that material. The region with low chromium concentration (Fig. 1c, black open circle) is characterized 
by luminescence at the wavelength of $473 \mathrm{~nm}$ (blue band) and a broad band with the maximum at the wavelength of $500 \mathrm{~nm}$ and with "tail" decay up to $620 \mathrm{~nm}$. The increase of chromium concentration (Fig. 1c, red triangle) leads to the less pronounced broad band. Simultaneously, the broad band is continuing up to $650-680 \mathrm{~nm}$, whereas the luminescence intensity is increased in the spectral range of 590-640 nm. Further increase of the chromium concentration (Fig. 1c, green open square) leads to suppression of the blue and broad bands, as well as appearance of a band with maximum at the wavelength of $560 \mathrm{~nm}$ (yellow band). The blue band associated with bound exciton is observed in all samples [8-10]. The broad band is not found in the spectra of the initial undoped samples (Fig. 1c, magenta solid square). However, a similar band with a shorter declining area (to the wavelength of 560-610 nm) is observed in the CVD-ZnSe annealed with HIP without doping (Fig. 1c, blue solid circle). The intensity of the band was reported to undergo changes only in the region of structural defects [8]. The initial peak analysis allows to propose that the broad band is a product of merging of lines at the wavelengths of 500, 520, $540 \mathrm{~nm}$ (green band), and 560-610 nm (yellow band).

Spatial distribution of luminescence intensity of all the above mentioned bands reveals a complex behavior. Figure 2 demonstrates planar maps and spatial dependences of luminescence intensity at the wavelengths of

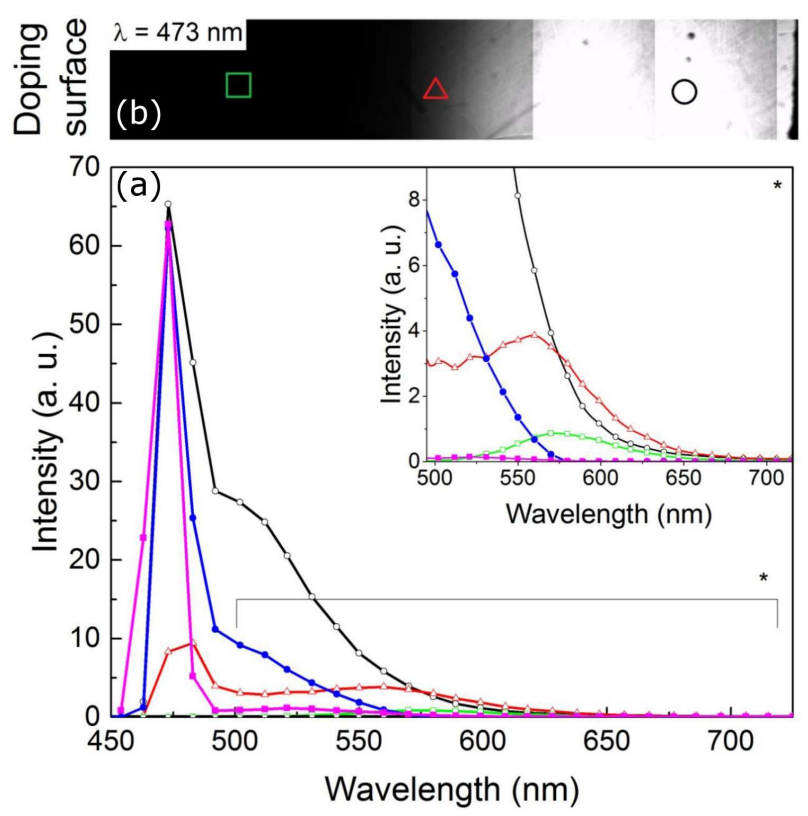

Fig. 1. (a) Two-photon excited luminescence spectra in regions with different concentrations of $\mathrm{Cr}$, marked at planar map (b): low concentration (black open circle), intermediate concentration (red triangle), high concentration (green open square) of chromium, and, in addition, the spectra of undoped both initial (magenta solid square) and annealed with HIP (blue solid circle) samples. Inset: enlarged spectral region of DIC-related emission. Spectra recorded at room temperature.

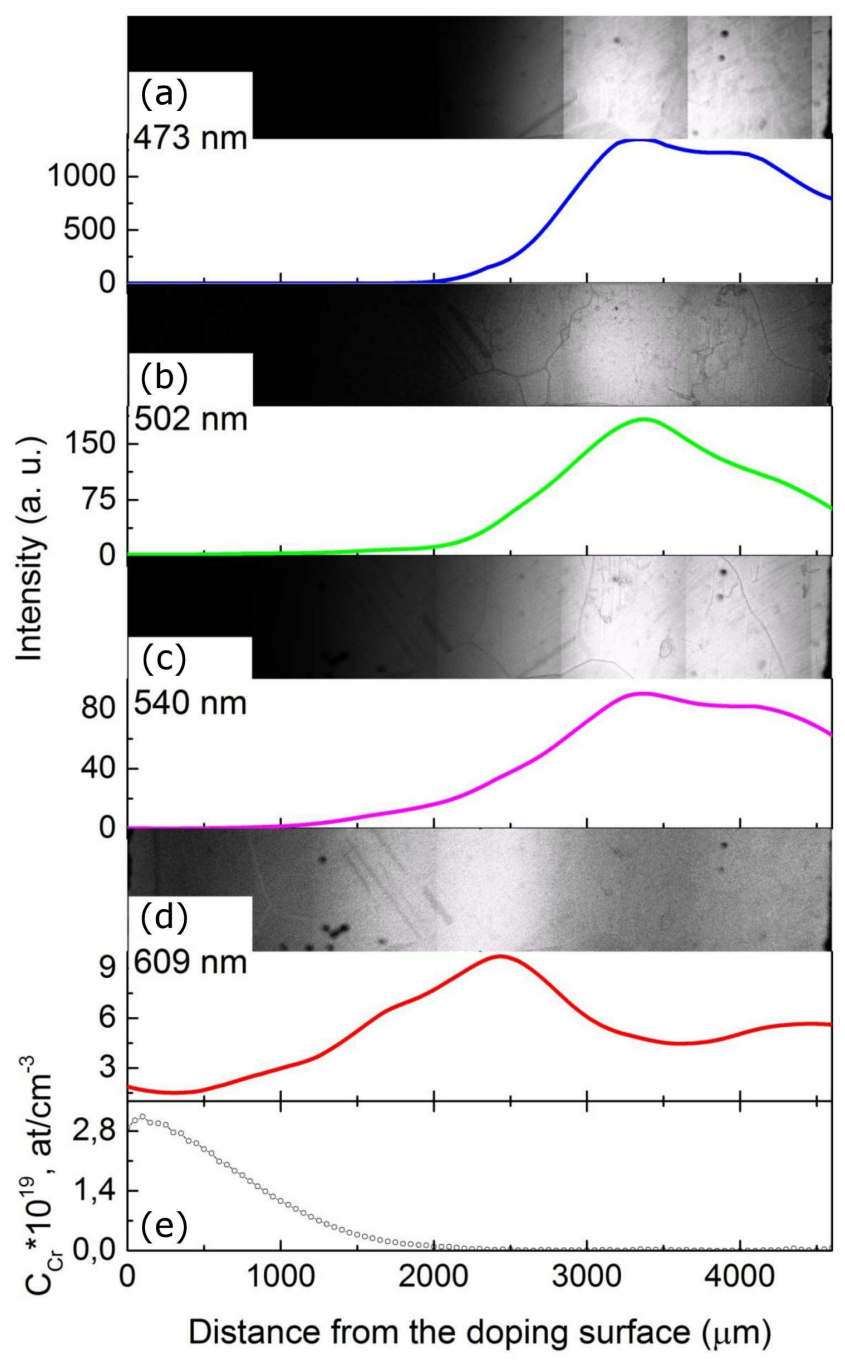

Fig. 2. Planar maps and spatial distributions of luminescence at wavelengths of 473, 502, 54 and $69 \mathrm{~nm}(\mathrm{a}-\mathrm{d})$ in $\mathrm{ZnSe}: \mathrm{Cr}$ crystal at room temperature accompanied with distribution of optically active $\mathrm{Cr}^{2+}(\mathrm{e})$.

$473,502,540$, and $609 \mathrm{~nm}$. The results are typical of the investigated samples. The following areas can be clearly identified: (1) the one with high chromium concentration (from the doping surface to $1.2 \mathrm{~mm}$ ); (2) the neighboring area with the chromium concentration in the range of $5 \times 10^{18} \mathrm{~cm}^{-3}$ to the detection threshold (from 1.2 to $2.5 \mathrm{~mm}$ from the surface); (3) the low (below the detection threshold) concentration area (from 2.5 to $4.2 \mathrm{~mm}$ from the surface); (4) the area at the crystal edge opposite to the doping surface with the chromium concentration of about $1-2 \times 10^{18} \mathrm{~cm}^{-3}$.

The area (1) is characterized by low intensity of the yellow band increasing with the decrease of chromium concentration attaining the maximum value at the distance of $2.3 \mathrm{~mm}$ from the doping surface, which is followed by the intensity decrease. As a result, a strip-like area with high intensity luminescence (AHIL) of the yellow 
band with the width of hundreds of $\mu \mathrm{m}$ and parallel to the doping surface is formed in the area (2) and parallel to the doping surface is formed in the area (2). Similar AHIL were previously observed in ZnSe:Fe crystals [7]. The blue and green bands increased in the area (2) and dominated in the area (3). In the area (4) these bands slightly decreased due to marginal chromium concentration, whereas the yellow band slightly increased.

We propose that green and yellow bands are results of doping procedure and are not related directly with dopant. Apparently, at least 2 types of defectiveimpurity centers (DICs) related to green and yellow bands are formed during doping. Different spatial distribution of green and yellow luminescence bands are allowed to suppose that related DICs are characterized by different diffusion coefficients. The green luminescence is distributed quite homogeneously in the crystal volume. This band was not observed in initial $\mathrm{ZnSe}$ and was detected in annealed with HIP samples both undoped and doped with Fe. Obviously, the annealing has an effect on whole crystal volume. Taking into account all the above, we suppose that nature of related DIC formation is caused by annealing process.

The yellow luminescence band was detected only in ZnSe doped with $\mathrm{Cr}$ and well recognized in area with high $\mathrm{Cr}$ concentration whereas the luminescence is suppressed by dopant; the suppression trend being well known [1214]. The spatial distribution of yellow band in $\mathrm{ZnSe}: \mathrm{Cr}$ is similar to spatial distribution of red band in ZnSe:Fe [10]. Both band demonstrated AHIL in area with high dopant concentration. Proceeding from this observation, we suppose that the nature of formation of related DICs are caused by doping process and not dependent on specific dopant. Probably, this trend is a general phenomenon and is applicable to a set of dopants in the A2B6 type semiconductors.

\section{Conclusion}

The chromium thermal-diffusion-based doping process of CVD-ZnSe at $1250^{\circ} \mathrm{C}$ leads to the formation of at least two types of DICs associated with the green (50$540 \mathrm{~nm}$ ) and yellow (560-610 nm) bands. DICs associated with the green band form due to annealing and are homogeneously distributed in the crystal volume. DICs associated with the yellow band are formed due to the chromium diffusion, the spatial distribution revealing a complex behavior. The AHIL are observed in the crystal regions with chromium concentration of $5 \times 10^{18}$ to $1 \times 10^{18} \mathrm{~cm}^{-3}$. Formation of strip-like AHIL parallel to the doping surface previously observed in $\mathrm{ZnSe}: \mathrm{Fe}$ is shown to be a general phenomenon rather than a specific property of particular crystals.

\section{Acknowledgments}

The reported study was funded by the Russian Science Foundation (Grant No. 19-13-00205 (development of technology and fabrication of $\mathrm{Cr}^{2+}: \mathrm{ZnSe}$ samples)) as well as by the Russian Foundation for Basic Research (Grant No. 18-29-20048 (the study of luminescence)). Part of the investigation was performed at The Center of Collective Use of Scientific Equipment of GPI RAS.

\section{References}

[1] S. Vasilyev, I. Moskalev, M. Mirov, V. Smolski, S. Mirov, V. Gapontsev, Opt. Mater. Expr. 7, 2636 (2017).

[2] P. Koranda, H. Jelínková, J. Šulc D.D.S., M. Nemec, M.E. Doroshenko, T.T. Basiev, V.K. Komar, A.S. Gerasimenko, V.M. Puzikov, Proc. SPIE 6451, 64510M (2007).

[3] S. Mirov, V. Fedorov, D. Martyshkin, I. Moskalev, M. Mirov, S. Vasilyev, IEEE J. Sel. Top. Quant. Electron. 21, 292 (2014).

[4] D.V. Savin, E.M. Gavrishchuk, V.B. Ikonnikov, O.N. Eremeykin, A.S. Egorov, Quant. Electron. 45, 8 (2015).

[5] J. Peppers, V.V. Fedorov, S.B. Mirov, Opt. Expr. 23, 4406 (2015)

[6] V.P. Kalinushkin, O.V. Uvarov, JETP Lett. 104, 754 (2016).

[7] V. Kalinushkin, O. Uvarov, A. Gladilin, J. Electron. Mater. 47, 5087 (2018).

[8] E.M. Gavrishchuk, A.A. Gladilin, V.P. Danilov, V.B. Ikonnikov, N.N. Il'ichev, V.P. Kalinushkin, A.V. Ryabova, M.I. Studenikin, N.A. Timofeeva, O.V. Uvarov, V.A. Chapnin, Inorg. Mater. 52, 1108 (2016).

[9] A.A. Gladilin, N.N. Ilichev, V.P. Kalinushkin, M.I. Studenikin, O.V. Uvarov, V.A. Chapnin, V.V. Tumorin, G.G. Novikov, Semiconductors 53, 1 (2019).

[10] S.S. Balabanov, E.M. Gavrischuk, A.A. Gladilin, V.B. Ikonnokov, N.N. Il'ichev, V.P. Kalinushkin, S.A. Mironov, D.V. Savin, M.I. Studenikin, N.A. Timofeeva, O.V. Uvarov, V.A. Chapnin, Inorg. Mater. 55, 459 (2019).

[11] T.V. Kotereva, V.B. Ikonnikov, E.M. Gavrishchuk, A.M. Potapov, D.V. Savin, Techn. Phys. 63, 1079 (2018).

[12] L.L. Kulyuk, R. Laiho, A.V. Lashkul, E. Lahderanta, D.D. Nedeoglo, I.V. Radevici, Physica B 405, 4330 (2010).

[13] M. Surma, M. Godlewski, T.R. Surkova, Phys. Rev. $B$ 50, 8319 (1994).

[14] M. Tabei, S. Shionoya, H. Ohmatsu, Jpn. J. Appl. Phys. 14, 240 (1975). 